\title{
BRUCK NETS WITH A TRANSITIVE DIRECTION*
}

To Helmut Salzmann on the occusion of his both hirthiay.

\begin{abstract}
We start the systematic investigation of the geometric properties and the collineation groups of Bruck nets $\mathbf{N}$ with a transitive direction (i.e. with a group $G$ of eentral transiations acting transitively on each line of a given parallel class $\mathbf{P}$ ). After reviewing some basic properties of such nets (in particular, their connection to difference matricesl, we shall consider the problem of what can be said if either $\mathbf{N}$ or $G$ admits an interesting extension. Specifically, we shall handle the following four situations: (1) there is a second transitive direction: (2) $\mathbf{N}$ is a translation net (w.l.o.g. with translation group $K$ containing $(G)$ : (3) the dual of $\boldsymbol{X} \mathbf{P}$ is a translation transversal design (w.l.o.g. with translation group $K$ containing $G$ ); (4) $\mathbf{N}$ admits a transversal fand can then in fact be extended by adding a further parallel class). Our study of these problems will yield interesting generalizations of known concepts (e.g. that of a fixed-point-free group automorphism) and results (for affine and projective planes). We shall also see that a wide variety of seemingly unrelated results and constructions scattered in the literature are in fact closeiy related and should be viewed as part of a unified whole.
\end{abstract}

\section{INTRODUCTION}

In this note, we shall start the systematic investigation of the geometric properties and the collineation groups of (finite) Bruck nets ${ }^{1} \mathbf{N}$ with a transitive direction (i.e. with a group $G$ of central translations acting transitively on each line of a given parallel class $\mathbf{P}$ ). Such nets generalize the notion of a projective plane with a $(p, L)$-transitivity for a flag $(p, L)$. After reviewing this result and some further basic properties of such nets (in particular, their connection to difference matrices and orthomorphisms). we shall consider the problem of what can be said if either $N$ or $G$ admits an interesting extension. Specifically, we shall study the following four situations:

(1) There is a second transitive direction.

(2) $\mathbf{N}$ is simultaneously a translation net.

\footnotetext{
*The results of this paper will form part of the first author's doctoral dissertation which is being written under the supervision of the second author.

All undefined terms will be explained in the following sections. As general background references, we mention Hughes and Piper [10] and Pickert [24] for the theory of projective planes and Beth et al. [3] for results from Design Theory and Finite Geomerries. An extensive survey on Latin squares, their geometries (i.e. nets and transversal designs) and their groups may be found in [20] where an overview of this very active area of study is given.
}

Geometriae Dedicata 36: 287-313, 1990.

C. 1990 Kluwer Academic Publishers. Printed in the Netherlands. 
(3) The dual of $\mathbf{N} \backslash \mathbf{P}$ is a translation transversal design.

(4) $\mathbf{N}$ admits a transversal (and can then in fact be extended by adding a further parallel class).

Our investigation of these problems will yield interesting generalizations of known concepts (e.g. that of a fixed-point-free group automorphism) and results (for affine and projective planes). It will turn out that the concept of central translations is much stronger than that of translations in general and might be viewed as the natural generalization of translations of affine planes to Bruck nets. We shall also see that a wide variety of seemingly little related results and constructions scattered in the literature are in fact closely connected and can be viewed as part of a unified whole by placing them in the context of the investigation of collineation groups of Bruck nets which admit sufficiently many central translations.

\section{CENTRAL TRANSLATIONS AND DIFFERENCE MATRICES}

In this section, we shall first review the basic material on Bruck nets with a transitive direction and their representation by difference matrices and, equivalently, by sets of pairwise orthogonal orthomorphisms which is fundamental for the remainder of this paper. Our presentation basically follows [18] (with some terminological changes introduced in [20]) where Theorem 2.3 below was first explicitly stated. One may, however, argue that these results are already implicit in [15], though in a rather disguised form. Following this, we shall give some new results on central translations.

We begin by recalling the definition of a Bruck net of order $s$ and degree $r$ (or, for short, an $(s, r)$-net). This is an incidence structure with parallelism $\mathbf{N}=(V, \mathbf{L}, \|)$ satisfying the following axioms:

(N1) Any two points are joined by at most one line.

(N2) Given any point $p$ and any line $L$, there is a unique line $L^{\prime}$ with $p \in L^{\prime} \| L$.

(N3) Any two non-parallel lines intersect (in a unique point, by (N1)).

(N4) There are $r \geqslant 3$ parallel classes each consisting of $s$ lines.

In the language of Design Theory, an $(s, r)$-net is the same as an affine 1-design $S_{r}\left(1, s, s^{2}\right)$. The dual of an $(s, r)$-net is called a transversal design (denoted a $\mathrm{TD}[r, s]$ ); in this setting, the parallel classes of lines of a net become the point classes of the TD. It is well known that an $(s, r)$-net is equivalent to $r-2$ mutually orthogonal Latin squares (MOLS) of order $s$. For details, the reader may consult [3] or [20].

Given any collineation $\gamma$ of a Bruck net $\mathbf{N}$, we call $\gamma$ a translation if $\gamma$ has no 
fixed points but fixes each parallel class; the identity is also considered a translation. We warn the reader that the product of two translations is not necessarily a translation again; we shall discuss examples for this later. We now recall the notion of a transitive direction for $\mathbf{N}$ :

2.1 DEFINITION. Let $\mathbf{P}$ be a parallel class of a Bruck net $\mathbf{N}$. We say that $\mathbf{N}$ is $\mathbf{P}$-transitive and call $\mathbf{P}$ a transitive direction for $\mathbf{N}$, if $\mathbf{N}$ admits a group $G$ of translations with the following two properties:

(2.1) Each element of $G$ fixes every line of $\mathbf{P}$.

(2.2) Given a line $L \in \mathbf{P}$ and two points $p$ and $q$ on $L$, there is a unique $\gamma \in G$ with $p^{\gamma}=q$.

It should be noted that Definition 2.1 generalizes the notion of $(p, L)$ transitivity in a projective plane $\Pi$ for a flag $(p, L)$. To see this, consider the affine plane $\mathbf{N}$ obtained from $\Pi$ by using the line $L$ as the line at infinity; then $\Pi$ is $(p, L)$-transitive if and only if the parallel class $\mathbf{P}$ of $\mathbf{N}$ determined by $p$ is a transitive direction for $\mathbf{N}$. Since $p$ is the center of the corresponding elations of $\Pi$, translations satisfying condition (2.1) for a suitable parallel class $\mathbf{P}$ will be called central translations with direction $\mathbf{P .}^{2}$ Note that, in general, a translation of a Bruck net need not be central. We shall discuss some important general results concerning central translations at the end of this section.

We next give the definition of two types of difference matrices:

2.2 DEFINITION. Let $G$ be an additively written (but not necessarily abelian) group of order $s$ and let $D=\left(d_{i j}\right)_{i=1, \ldots, r ; j=1 \ldots, s}$ be a matrix with entries from $G$. We call $D$ a left $(s, r)$-difference matrix over $G$, if the following condition is satisfied:

$$
\begin{aligned}
& \left\{-d_{i j}+d_{h j}: j=1, \ldots, s\right\}=G \text { for any two distinct indices } \\
& h, i \in\{1, \ldots, r\} .
\end{aligned}
$$

In other words, the 'left' differences of corresponding entries of any two rows in $D$ contain each element of $G$ exactly once. Similarly, we shall call $D$ a right $(s, r)$-difference matrix if it satisfies the condition

$$
\begin{aligned}
& \left\{d_{i j}-d_{h j}: j=1, \ldots, s\right\}=G \text { for any two distinct indices } \\
& h, i \in\{1, \ldots, r\}
\end{aligned}
$$

Of course, it makes no essential difference which one of these conditions is considered since replacing $D$ by $-D$ interchanges the two species of difference

${ }^{2}$ In [18] and [23], the term 'strict translations' was used instead. 
matrices defined by $(2.3)$ and $\left(2.3^{\prime}\right)$, respectively. In the abelian case, both conditions are equivalent, anyway. Thus we may suppress the specification 'left' and 'right', respectively, if we are in the abelian case or if we only talk about questions of existence. Usually, the term 'difference matrix' in the literature means 'right difference matrix' as defined here. In what follows, we shall indeed need both types of difference matrices.

The following fundamental result on the equivalence of difference matrices and Bruck nets with a transitive direction was first explicitly stated in [18]; since it is crucial for all that follows, we shall include its proof.

2.3 THEOREM. The existence of an $(s, r)$-difference matrix $D$ over $G$ is equivalent to that of an $(s, r+1)$-net $\mathbf{N}$ admitting $G$ as a transitive group of central translations ( for a suitable direction $\mathbf{P}$ ).

Proof. First, let $D$ be a left $(s, r)$-difference matrix over $G$. We construct $\mathbf{N}$ as follows: As point set, we choose $V=\{(j, x): j=1, \ldots, s ; x \in G\}$. We then define parallel classes of lines $\mathbf{P}_{0}=\left\{L_{j}: j=1, \ldots, s\right\}$ and $\mathbf{P}_{i}=\left\{L_{i, x}: x \in G\right\}$ for $i=1, \ldots, r$, where $L_{j}=\{(j, x): x \in G\}$ and $L_{i, x}=\left\{\left(j, d_{i j}+x\right): j=1, \ldots, s\right\}$. It is then clear that the $\mathbf{P}_{i}(i=0, \ldots, r)$ are indeed parallel classes of lines and that $G$ acts on $\mathbf{N}$ as a group of central translations: The element $g \in G$ maps $(j, x)$ to $(j, x+g)$, fixes each line in $\mathbf{P}_{0}$ and maps $L_{i, x}$ to $L_{i, x+g}$; hence $\mathbf{N}$ is $\mathbf{P}_{0^{-}}$ transitive. It only remains to check that $\mathbf{N}$ is indeed a Bruck net, which requires proving that any two non-parallel lines intersect in a unique point. This is clear if one of the lines belongs to $\mathbf{P}_{0}$; thus consider any two lines $L_{i, x}$ and $L_{h, y}$ where $h \neq i$ and note that a point $(j, z)$ is on both lines if and only if we have $z=d_{i j}+x=d_{h j}+y$. This condition is equivalent to the equations $z=d_{i j}+x$ and $-d_{h j}+d_{i j}=y-x$; the second of these equations has a unique solution for $j$ by (2.3), and then $z$ is determined from the first equation.

Conversely, if $\mathbf{N}$ is an $(s, r+1)$-net with $G$ as a transitive group of central translations with direction $\mathbf{P}_{0}$, we can choose a 'base point' $p_{j}$ on each of the lines $L_{j}$ in $\mathbf{P}_{0}$; the condition (2.2) allows us to identify each point $p_{j^{\prime}}$ of $L_{j}$ with the pair $(j, x)$ where $x$ is the unique element of $G$ which maps $p_{j}$ to $p_{j^{\prime}}$. We then choose a 'base line' $L_{i, 0}$ in each of the remaining parallel classes $\boldsymbol{P}_{i}$ $(i=1, \ldots, r)$ and define $D$ by writing $L_{i, 0}=\left\{\left(j, d_{i j}\right): j=1, \ldots, s\right\}$, where $\left(j, d_{i j}\right)$ is the point of intersection of $L_{i, 0}$ with $L_{j}$. As $G$ fixes each parallel class, we see at once that we have $\mathbf{P}_{i}=\left\{L_{i, x}: x \in G\right\}$, where $L_{i, x}=\left\{\left(j, d_{i j}+x\right): j=1, \ldots, s\right\}$. The argument that $D$ is a left difference matrix is now just the converse of the computation in the first part of the proof.

We remark that we have chosen $D$ to be a left difference matrix in the proof of Theorem 2.3 in order to make sure that we can have $G$ act by right 
translation $^{3}$ (which is both customary and preferable in studying the connections to translation nets later). If we had considered right difference matrices instead, we would then have to let $G$ act by left translation. However, in the dual setting of transversal designs in Section 5, we shall need right difference matrices for similar reasons.

We mention that there are two other conditions which are equivalent to the situation of Theorem 2.3 (cf. Pickert [25]). The first of these is in terms of Latin squares: $\mathbf{N}$ belongs to a set of MOLS one of which is the group table of $G$, while the remaining Latin squares arise from this group table by suitable column permutations. The second condition is in terms of configurations: $\mathbf{N}$ satisfies the Reidemeister condition with respect to $\mathbf{P}$ and any two other parallel classes as well as the Desargues condition with respect to $\mathbf{P}$. We shall not make use of these results.

Difference matrices have first been explicitly used by Bose and Bush [5] for the construction of sets of MOLS; the term 'difference matrix' was introduced in [15]. They are equivalent to the 'orthomorphism method' for constructing MOLS, which is due to Mann [21] (who rather uses the equivalent terminology of 'complete mappings') and to Johnson et al. [12]. Since it will be very convenient to use the language of orthomorphisms later, we shall recall the required definitions; again, we shall need to distinguish two species of orthomorphisms.

2.4 DEFINITION. A left orthomorphism of a group $G$ is a permutation $\varphi$ for which the mapping $g \rightarrow-g+\varphi(g)$ is also a permutation of $G$. Two left orthomorphisms $\theta$ and $\varphi$ are called orthogonal if the mapping $g \rightarrow-\varphi(g)+\theta(g)$ is also a permutation of $G$. Similarly, $\varphi$ is called a right orthomorphism of $G$ if the mapping $g \rightarrow g-\varphi(g)$ is a permutation; two right orthomorphisms $\theta$ and $\varphi$ are called orthogonal if the mapping $g \rightarrow \varphi(g)-\theta(g)$ is also a permutation of $G$.

2.5 LEMMA. Let $G$ be a group of order $s$. Then the existence of a left $(s, r)$ difference matrix over $G$ is equivalent to that of $r-2$ pairwise orthogonal left orthomorphisms of $G^{4}{ }^{4}$

Proof. Let $D$ be a left difference matrix which is w.l.o.g. normalized, i.e. $D$ has first row and first column containing entries 0 only (which may be

${ }^{3}$ In [3] and [18] we have, unfortunately, made a mistake by using right difference matrices where we should have used left difference matrices.

${ }^{4}$ Evans [9] considers the orthomorphism graph $\operatorname{Orth}(G)$ of $G$, the vertices of which are the left orthomorphisms with two orthomorphisms being adjacent if and only if they are orthogonal. Thus a left $(s, r)$-difference matrix over $G$ is equivalent to a clique of size $r-2$ in $\operatorname{Orth}(G)$. 
achieved by suitable left additions to the columns and right additions to the rows). We then see that we may consider rows $2, \ldots, r$ as permutations of $G$, where row 2 is taken to be the identity permutation (i.e. we may index the columns of $D$ by their entries in the second row). Then condition (2.3) for rows 2 and $i \geqslant 3$ just means that the permutation belonging to row $i$ is a left orthomorphism which will be denoted by $\varphi_{i}$. Condition (2.3) for rows $h$ and $i$ (with $h, i \geqslant 3$ and $h \neq i$ ) thus just means that the corresponding orthomorphisms $\varphi_{h}$ and $\varphi_{i}$ are orthogonal. The converse is similar.

The fact that a left difference matrix $D$ determines a net $\mathbf{N}(D)$ thus just corresponds to the well-known result that the existence of $t$ pairwise orthogonal orthomorphisms of $G$ implies that of $t+1$ MOLS of order $|G|$. Orthomorphisms have been studied for a long time; some of our results below will then generalize known theorems on affine planes described by complete sets of pairwise orthogonal orthomorphisms (i.e. $t=|G|-2$ ) or, equivalently, Cartesian groups to Bruck nets.

We now give a slightly different description of Bruck nets with a transitive direction using orthomorphisms:

2.6 REMARK. As in the proof of 2.5, we index the columns of the normalized left difference matrix $D$ by the elements of $G$ (using the second row). Then we may change our coordinatization of the associated Bruck net correspondingly: We now identify the point set with $G \times G$ and label the lines of $\mathbf{P}_{0}$ as $L_{g}=\{(g, z): z \in G\}(g \in G)$; as $D$ is normalized, the lines in $\mathbf{P}_{1}$ are the $L_{1 . z}=\{(g, z): g \in G\}$. Then the remaining lines may be written in the form $L_{i . z}=\left\{(x, y) \in G \times G: \varphi_{i}(x)+z=y\right\}$ (with $i=2, \ldots, r$ and $\left.z \in G\right\}$ ) which looks quite as in the classical case of affine planes over a field (where, of course, the corresponding orthomorphisms are induced by multiplication with suitable elements). This is the notation used by Evans [9]. In Section 4, we shall require a slight variation of this representation.

We close this section with a few simple but important geometric results which seem to be new. They show that central translations may be considered to be the natural generalization of the concept of translations from affine planes to Bruck nets. Note that general translations of Bruck nets may behave rather strangely; for instance, there may be more than one translation mapping a point $p$ to a given other point $q$. Examples for this situation are contained in the work of Sprague [30]. However, central translations cannot behave like this: 
2.7 PROPOSITION. Let $p$ and $p^{\prime}$ be two points of a Bruck net $\mathbf{N}$. Then there exists at most one central translation $\gamma$ with $p^{\prime \prime}=p^{\prime} .^{5}$

Proof. Note first that the existence of a central translation $;$ with $p^{\gamma}=p^{\prime}$ implies that $p$ and $p^{\prime}$ lie on a common line $L$. Consider any point $q$ not on $L$ which is joined to $p$ by a line $M$, and denote by $L^{\prime}$ and $M^{\prime}$ the parallels to $L$ and $M$ through $q$ and $p^{\prime}$, respectively. Since $\gamma$ is a central translation, $q^{\gamma}$ is the point of intersection of $M^{\prime}$ and $L^{\prime}$. Thus the images of points joined to $p$ are uniquely determined by $p^{\prime}$. Now let $x$ be any point distinct from $p$ on $L$. Since $r \geqslant 3$ by definition of a Bruck net, we may choose non-parallel lines $M$ and $N$ distinct from $L$ through $p$ and $x$, respectively. Then $M$ and $N$ intersect in a point $q$, and - as we have seen $-p^{\prime}$ determines the image $q^{\prime}$ of $q$; then, using the same argument, $q^{\prime}$ determines the image $x^{\prime}$ of $x$. Thus $y^{\prime}$ is also determined for all points on $L$. It is now easily seen that $\gamma$ is uniquely determined for every point.

2.8 LEMMA. Let $\mathbf{N}$ be a Bruck net, let $\gamma$ be a central translation with direction $\mathbf{P}$ and let $\delta$ be a 'dilatation' (i.e. any collineation fixing all parallel classes of $\mathbf{N}$ ). Then $\delta^{-1} \gamma \delta$ is also a central translation with direction $\mathbf{P}$.

Proof. It is clear that $\delta^{-1} \gamma \delta$ fixes every line in $\mathbf{P}$. If $\delta^{-1} \gamma \delta$ has a fixed point $p$, the proof of 2.7 above shows that $\delta^{-1} \gamma \delta=\mathrm{id}$. (Note that we did not use the fact that $p^{\prime} \neq p$.) Thus $\delta^{-1} \gamma \delta$ is either fixed-point-free or the identity, i.e. a (central) translation.

It should be noted that dilatations can behave quite strangely, too; for instance, a non-identity dilatation may have several fixed points. The reader is invited to construct examples for this situation. Trivially, all dilatations of a Bruck net $\mathbf{N}$ form a group $D$. Then we have the following consequence of the two previous results:

2.9 COROLLARY. Let $\mathbf{N}$ be a Bruck net which is transitive in direction $\mathbf{P}$, and denote the corresponding group of central translations by $G$. Then $G$ is a normal subgroup of the group of dilatations of $\mathbf{N}$.

Proof. As usual, denote the order of $\mathbf{N}$ by $s$; thus $G$ consists of $s$ central translations with direction $\mathbf{P}$. Now let $\delta$ be any dilatation of $\mathbf{N}$. By Lemma $2.8, \delta^{-1} G \delta$ also consists of $s$ central translations with direction $\mathbf{P}$. But by Proposition 2.7, there are at most $s$ central translations with direction $\mathbf{P}$. Thus we have $\delta^{-1} G \delta=G$.

\footnotetext{
5 In the next few results, we view the groups considered as permutation groups; thus we shall now write groups multiplicatively and denote images using exponential notation.
} 


\section{BRUCK NETS WITH TWO TRANSITIVE DIRECTIONS}

In this section we consider Bruck nets admitting at least two transitive directions. Here we have the following fundamental result:

3.1 THEOREM. Let $\mathbf{N}$ be a Bruck net which has two transitive directions $\mathbf{P}$ and $\mathbf{Q}$, and denote the corresponding transitive groups of central transiations by $G$ and $H$, respectively. Then the collineation group $K$ generated by $G$ and $H$ is the direct product $G \times H$. Moreover, $K$ consists of translations of $\mathbf{N}$ and acts regularly on the point set of $\mathbf{N}$.

Proof. It is clear that one has $|G \cap H|=1$. Thus the assertion is a simple consequence of Corollary 2.9 which shows that both $G$ and $H$ are normal subgroups of $K$ which obviously acts regularly on the point set of $\mathbf{N}$ and consists of translations of $\mathbf{N}$.

We emphasize that a net with two transitive directions does not necessarily have all its parallel classes as transitive directions, even though the group $K=G \times H$ acts as a transitive group of translations. This is due to the fact that the remaining translations in $K$ need not be central. To discuss this problem in its proper setting, we have to recall the notion of a translation net as introduced in [16] and [30].

3.2 DEFINITION. Let $K$ be a collineation group of a Bruck net $\mathbf{N}$ which acts regularly on the set of points and fixes each parallel class. Then $\mathbf{N}$ is called a translation net with translation group $K$.

In [30], Sprague gave examples for the fact that a Bruck net may be a translation net with respect to different, even non-isomorphic, translation groups. Thus a translation net should always be considered as a pair $(\mathbf{N}, K)$. Translation nets can be described by the following generalization of Andre's [1] congruence partitions:

3.3 DEFINITION. Let $K$ be a group of order $s^{2}$ and let $U_{1}, \ldots, U_{r}$ (with $r \geqslant 3$ ) be pairwise disjoint subgroups of order $s$, i.e. subgroups satisfying $U_{i} \cap U_{j}=\{0\} \quad$ (or, equivalently, $U_{i}+U_{j}=K$ ) whenever $i \neq j$. Then $\mathbf{U}=\left\{U_{1}, \ldots, U_{r}\right\}$ is called a partial congruence partition of order $s$ and degree $r$ (or, for short, an $(s, r)-P C P$ ) with components $U_{1}, \ldots, U_{r}$.

3.4 PROPOSITION. Let $\mathrm{U}$ be an $(s, r)-P C P$ in the group $K$. Then the incidence structure

$$
\mathbf{N}=\mathbf{D}(\mathbf{U})=\left(K,\left\{U_{i}+x: i=1, \ldots, r ; x \in K\right\}, \epsilon\right)
$$

is an $(s, r)$-translation net with translation group $K$. Moreover, every translation net may be represented in this way. 
The simple proof (which may be found in [3], [16] or [30]) will be left to the reader. We just sketch how one constructs the PCP from the net: One identifies the point set of $N$ with $K$ and takes as the $U_{i}$ the stabilizers of the lines through the point 0 . In this terminology, Theorem 3.1 says that a Bruck net with two transitive directions $\mathbf{P}$ and $\mathbf{Q}$ is a translation net with a translation group $K$ which is the direct product of the two transitive groups $G$ and $H$ of central translations (which are, of course, among the $U_{i}$, since they are the stabilizers of the lines in $\mathbf{P}$ and $\mathbf{Q}$, respectively). For obvious reasons, such a translation net is called a splitting translation net (cf. [2]). In this connection, we note the following interesting (but simple) results of Sprague [30] on the structure of translation nets; part (a) is in fact obvious and proofs for parts (b) and (c) will be a by-product of work in Section 4.

3.5 PROPOSITION. Let $\mathbf{N}$ be a translation $(s, r)$-net with translation group $K$, described by the PCP U. Then one has the following:

(a) $U_{i}$ is a normal subgroup of $K$ if and only if $U_{i}$ consists of central translations.

(b) If $U_{1}$ is a normal subgroup, then the remaining components $U_{2}, \ldots, U_{r}$ are all isomorphic.

(c) If both $U_{1}$ and $U_{2}$ are normal subgroups, then all components are isomorphic and one has $K=U_{1} \times U_{2}$.

(d) If three components of $\mathbf{U}$ are normal, then $K$ is abelian and thus all components are normal.

Sprague [30] gives examples showing that all the cases mentioned in 3.5 can actually occur. As a consequence of 3.5 , we have the following result:

3.6 COROLLARY. Let $\mathbf{N}$ be a Bruck net with two transitive directions, as in Theorem 3.1. Then the groups $G$ and $H$ are isomorphic.

We have now seen that Bruck nets with two transitive directions are equivalent to splitting translation nets. Recently, these have been studied extensively by Bailey and Jungnickel [2]. Evans [9] has studied the same problem in a different but equivalent formulation. We shall once more return to splitting translation nets in the next section where we study a more general situation.

\section{TRANSLATION NETS WITH a TRANSITIVE DIRECTION}

In this section we shall consider the connection between translation nets and nets with a transitive direction. We first show that the corresponding PCPs 
allow the construction of a collection of difference matrices: this result is basicaliy contained in [16].

4.1 PROPOSITION. Let $G$ be a component of an (s.r)-PCP $\mathbf{U}$ in the group $K$. Then there exists an $(s, r-1)$-difference matrix over $G$.

Proof. Let $\mathbf{U}=\left\{U_{1}, \ldots, U_{r}\right\}$ and assume w.l.o.g. that $G=U_{r}$. We shall give an explicit construction for a left $(s, r-1)$-difference matrix $D$ over $G$. Put $H=U_{1}$ and note that every element $k \in K$ has a unique representation $k=h+g$ with $g \in G$ and $h \in H$. In particular, every $\mathscr{h}_{i} \in U_{i}(i=1, \ldots, r-1)$ has a unique representation in the form $y_{i}=h+\varphi_{i}(h)$ with $h \in H$ and $\varphi_{i}(h) \in G$. As each $U_{i}$ is a system of coset representatives for $G$, this defines $r-1$ mappings $\varphi_{i}: H \rightarrow G(i=1, \ldots, r-1)$. We now construct a matrix $D=\left(d_{i h}\right)_{i=1, \ldots, r-1: h \in H}$ over $G$ by putting $d_{i n}:=\varphi_{i}(h)$ and claim that $D$ is a left difference matrix. To see this, consider two different rows $i$ and $j$ of $D$ and assume that

$$
-\varphi_{i}(x)+\varphi_{j}(x)=-\varphi_{i}(y)+\varphi_{j}(y) \text { for } x, y \in H
$$

we have to show that this implies $x=y$. By definition of the $\varphi_{i}(h)$, there are elements $u, u^{\prime} \in U_{i}$ and $v, v^{\prime} \in U_{j}$ satisfying $x+\varphi_{i}(x)-u, y+\varphi_{i}(y)=u^{\prime}$, $x+\varphi_{j}(x)=v$ and $y+\varphi_{j}(y)=v^{\prime}$. Thus $\left(^{*}\right)$ implies $(-u+x)+(-x+v)$ $=\left(-u^{\prime}+y\right)+\left(-y+v^{\prime}\right)$ and thus $u^{\prime}-u=v^{\prime}-v \in U_{i} \cap U_{j}=\{0\}$ which gives $u=u^{\prime}$ and $v=v^{\prime}$. We now have $x+\varphi_{i}(x)=u=y+\varphi_{i}(y)$ and thus $\varphi_{i}(y)-\varphi_{i}(x)=-y+x \in G \cap H=\{0\}$ which finally proves that indeed $x=y$.

We note that the mappings $\varphi_{i}$ in the preceding proof have the following properties:

(4.1) $\varphi_{1}(h)=0$ for all $h \in H$;

(4.2) $\varphi_{i}$ is a bijection fixing 0 for $i=2, \ldots, r-1$;

(4.3) For any two distinct indices $i, j \in\{1, \ldots, r-1\}$, the mapping $h \rightarrow-\varphi_{i}(h)+\varphi_{j}(h)$ is also a bijection.

Since this would for $G=H$ be the properties of pairwise orthogonal left orthomorphisms, we introduce the following terminology:

4.2 DEFINITION. Let $G$ and $H$ be two groups of order $s$. Any bijection $\varphi: H \rightarrow G$ will be called a $(G, H)$-orthomorphism; two $(G, H)$-orthomorphisms $\varphi$ and $\theta$ will be called orthogonal, if the mapping $h \rightarrow-\varphi(h)+\theta(h)$ is also a bijection.

4.3 REMARK. Let $G$ and $H$ be any two groups of order $s$. Then a normalized left $(s, r)$-difference matrix $D$ over $G$ and a system of $r-1$ pairwise orthogonal $(G, H)$-orthomorphisms $\Phi=\left\{\varphi_{2}, \ldots, \varphi_{r}\right\}$ are equivalent objects. (Given $D$, the required $(G, H)$-orthomorphisms can be obtained in the 
obvious way after arbitrarily indexing the columns of $D$ with the elements of $H$.) We may use this to give a final alternative description of the corresponding $(s, r+1)$-net which generalizes that of Evans [9] discussed in Remark 2.6: We now identify the point set with the set $H \times G$ and label the lines of $\mathbf{P}_{0}$ as $L_{i h}=\left\{(h, g): g \in G_{j} \quad(h \in H)\right.$; as $D$ is normalized, the lines in $\mathbf{P}_{1}$ are the $L_{1 . z}=\{(h, z): h \in H\}(z \in G)$. The remaining lines may be written in the form $L_{i, z}=\left\{(h, g) \in H \times G: \varphi_{i}(h)+z=g\right\}(i=2, \ldots, r ; z \in G)$.

The following geometric result is an immediate consequence of 2.3, 3.4 and 4.1:

4.4 COROLLARY. The existence of a translation ( $s, r)$-net implies that of an $(s, r)$-net with a transitive direction.

We shall consider the situation of Corollary 4.4 a little more closely and show the following:

4.5 PROPOSITION. Let $G=U_{r}$ be a component of an $(s, r)-P C P \mathbf{U}$ in the group $K$ and let $\mathbf{N}$ be the corresponding translation net. Put $H=U_{1}$ and construct the left $(s, r-1)$-difference matrix $D$ over $G$ as in the proof of Proposition 4.1. Finally, denote the ( $s, r)$-net corresponding to $D$ (as described in Remark 4.3) by $\mathbf{M}$. Then $\mathbf{N}$ is isomorphic to $\mathbf{M}$ if and only if $G$ is a normal subgroup of $K$.

Proof. We use the fact that every element $k \in K$ has a unique representation $k=h+g$ with $g \in G$ and $h \in H$ to identify $K$ with the set $H \times G=\{(h, g): h \in H$ and $g \in G\}$. Using the notation of the proof of 4.1 and of 4.3 , we now have

$$
U_{i}+z=\left\{\left(h, \varphi_{i}(h)+z\right): h \in H\right\}=L_{i, z}
$$

for $i=1, \ldots, r-1$ and $z \in G$. Thus the two nets $\mathbf{N}$ and $\mathbf{M}$ have the $r-1$ parallel classes $\mathbf{P}_{1}, \ldots, \mathbf{P}_{r-1}$ in common. Returning to standard notation and identifying the point set with $K$ again, we see that $\mathbf{M}$ contains the further parallel class $\mathbf{P}_{0}=\left\{L_{h}: h \in H\right\}$ with $L_{h}=\{(h, g): g \in G\}=h+G$, and $\mathbf{N}$ contains the further parallel class $\vec{P}_{r}=\{G+h: h \in H\}$. Clearly, these two parallel ciasses agree if and only if $G$ is a normal subgroup of $K$.

In view of Proposition 4.5, it will be natural to consider PCPs with a normal component. Geometrically, these correspond to Bruck nets $\mathbf{N}$ with a transitive direction which are simultaneously translation nets. Denoting the translation group by $K$ and the transitive group of central translations by $G$, we have $G \triangleleft K$ in the situation of 4.5 . However, this is not necessarily true in general-not even when $\mathbf{N}$ has all its directions transitive. The following 
example due to Sprague [30] illustrates this: The translation $(6,3)$-net $\mathbf{N}$ with translation group $\mathbf{Z}_{6} \times \mathbf{Z}_{6}$ and components $\{0\} \times \mathbf{Z}_{6}, \quad \mathbf{Z}_{6} \times\{0\}$ and $\left\{(z, z): z \in \mathbf{Z}_{6}\right\}$ with three transitive directions can also be represented as a translation net with a non-abelian translation group $K$ with only one transitive direction. For details, the reader is referred to [30]. A further example for this situation will be given in 6.7 below. Nevertheless, the following result shows that we may w.l.o.g. assume that there arises the more pleasant situation where $G$ is contained in $K$.

4.6 PROPOSITION. Let $\mathbf{N}$ be a translation net with translation group $K$ and assume that $\mathbf{N}$ is also $\mathbf{P}$-transitive for some direction $\mathbf{P}$, with corresponding group $G$ of central translations. Then $\mathbf{N}$ is also a translation net for some translation group $K^{\prime}$ containing $G$ as a normal subgroup.

Proof. Let $\mathbf{N}$ be represented by the partial congruence partition $\mathbf{U}$ in $K$ (as in 3.4). If $G$ is one of the components of $U$, there is nothing to prove. Thus assume the contrary, and let $U$ be the component of $\mathbf{U}$ corresponding to the line $L$ of $\mathbf{P}$ through the origin $O$. Choose any other component $V$ (corresponding to the line $M$ through $O$ ) of $\mathbf{U}$ and consider the group $K^{\prime}$ generated by $G$ and $V$. Clearly $K^{\prime}$ has order at most $s^{2}$ (since both $G$ and $V$ have order $s$ ) and consists of dilatations of $\mathbf{N}$. By Corollary 2.9, $G$ is a normal subgroup of $K^{\prime}$. Also, given any point $p$ of $\mathbf{N}$, there is an element of $K^{\prime}$ mapping $O$ to $p$ : To see this, note first that the lines in the parallel class $\mathbf{P}$ are the images of $L$ under the elements of $V$, since the $s$ translations in $V$ clearly act transitively on the $s$ lines in $\mathbf{P}$. Thus let $L^{\prime}$ be the line through $p$ in $\mathbf{P}$ and choose $\delta \in V$ with $L^{\prime}=L^{\delta}$. Then $O^{\delta}$ lies on $L^{\prime}$, and since $G$ is a transitive group of central translations with direction $\mathbf{P}$, there exists $\gamma \in G$ with $O^{\delta y}=p$. Thus $K^{\prime}$ contains at least $s^{2}$ translations. It follows that $K^{\prime}$ has order $s^{2}$ and acts regularly on the point set of $\mathbf{N}$, which means that $K^{\prime}$ is a translation group for $\mathbf{N}$.

Thus we may assume from now on that $G$ is a normal subgroup of $K$ whenever we study a Bruck net $\mathbf{N}$ with a transitive group $G$ of central translations which is simultaneously a translation net with translation group $K$. In the previous section, we have already considered the special case where $\mathrm{N}$ actually admits a second transitive group $H$ of central translations; as we have seen, we may then even assume $K=G \times H$. We shall now study the case where $K$ does not necessarily split as a direct product. This will result in an interesting generalization of the notion of fixed-point-free group automorphisms. We first introduce the following generalization of group homomorphisms: 
4.7 DEFINITION. Let $G$ and $H$ be two groups, and let $\pi: H \rightarrow$ Aut $G$ be a homomorphism. A mapping $\varphi: H \rightarrow G$ is called a semi-homomorphism (with companion homomorphism $\pi$ ) if it satisfies the following condition:

$$
\varphi\left(h+h^{\prime}\right)=\pi_{h^{\prime}}(\varphi(h))+\varphi\left(h^{\prime}\right),
$$

where we write $\pi_{h}$ for the automorphism $\pi(h)$ of $G$. A bijective semihomomorphism will be called a semi-isomorphism.

Note that (4.4) reduces to the definition of a homomorphism in the trivial case where $\pi_{h}=\mathrm{id}_{G}$ for all $h \in H$. In order to explain the meaning of semihomomorphisms, we recall that we may use $\pi$ to form a semi-direct product $K$ of $G$ by $H$ as follows (see, e.g., Huppert [11]): $K$ is defined on the set $H \times G$ with addition

$$
(h, g)+{ }_{\pi}\left(h^{\prime}, g^{\prime}\right)=\left(h+h^{\prime}, \pi_{h^{\prime}}(g)+g^{\prime}\right) ;
$$

for the trivial choice of $\pi$, this gives of course the direct sum $G \oplus H$. We shall now show that semi-homomorphisms are equivalent to subgroups $U$ of $K$ of the same order as $H$ which intersect the subgroup $G^{\hat{}}=\{0\} \times G$ trivially:

4.8 LEMMA. Let $G$ and $H$ be two finite groups, let $\pi: H \rightarrow$ Aut $G$ be a homomorphism, and denote the corresponding semi-direct product of $G$ by $H$ as $K$. Finally, let $\varphi: H \rightarrow G$ be a semi-homomorphism. Then $U_{\varphi}=\{(h, \varphi(h)): h \in H\}$ is a subgroup of $K$ of order $|H|$ with $|U \cap \hat{G}|=1$; moreover, $U_{\varphi}$ is isomorphic to $H$. Conversely, any subgroup $U$ of order $|H|$ intersecting $G^{+}$trivially can be represented in this way.

Proof. We first note that a semi-homomorphism satisfies $\varphi(0)=0$; this is easily seen by putting $h=0$ in (4.4). Thus $U_{\varphi}$ contains $(0,0)$. It now suffices to show that $U_{\varphi}$ is closed under addition. But in view of (4.5) this requirement just reduces to the defining condition (4.4) for a semi-homomorphism. Conversely, let $U$ be a subgroup of $K$ of order $|H|$ with $\left|U \cap \hat{G^{\wedge}}\right|=1$. For reasons of cardinality, $U$ intersects each coset of $G^{\wedge}$ in exactly one element. Since these cosets are the sets $(h, 0)+\widehat{G^{\prime}}$, we may write $U=\{(h, \varphi(h)): h \in H\}$ for a suitable mapping $\varphi: H \rightarrow G$. Using (4.5) and the fact that $U$ is closed under addition, we obtain the validity of (4.4). It remains to show that $U_{\varphi}$ is indeed isomorphic to $\mathrm{H}$. Define $\alpha: H \rightarrow U_{\varphi}$ by $\alpha(h):=(h, \varphi(h))$. Then $\alpha$ is an isomorphism, since we have

$$
\begin{aligned}
\alpha\left(h+h^{\prime}\right) & =\left(h+h^{\prime}, \varphi\left(h+h^{\prime}\right)\right)=\left(h+h^{\prime}, \pi_{h^{\prime}}(\varphi(h))+\varphi\left(h^{\prime}\right)\right) \\
& =(h, \varphi(h))+\left(h^{\prime}, \varphi\left(h^{\prime}\right)\right)=\alpha(h)+\alpha\left(h^{\prime}\right) .
\end{aligned}
$$

We can now prove the following result characterizing the PCPs with a normal component: 
4.9 PROPOSITION. Let $G$ and $H$ be two groups of order s, let $\pi: H \rightarrow$ Aut $G$ be a homomorphism and denote the corresponding semi-direct product of $G$ by $H$ as $K$. Finally, let $\Phi=\left\{\varphi_{2}, \ldots, \varphi_{r-1}\right\}$ be a system of pairwise orthogonal semiisomorphisms from $H$ onto $G$. Then the corresponding $(s, r)$-net $\mathbf{N}$ (cf. Remark $4.3)$ is a translation net with translation group $K$. Moreover, $\mathbf{N}$ belongs to the $P C P \quad \mathbf{U}=\left\{U_{1}, \ldots, U_{r}\right\}$, where $U_{1}=H \times\{0\}, U_{r}=\{0\} \times G$ and $U_{i}=$ $\left\{\left(h, \varphi_{i}(h)\right): h \in H\right\}$ for $i=2, \ldots, r-1$. Conversely, every translation net with a transitive direction may be obtained in this way.

Proof. We see from Remark 4.3 that we have $U_{1}=L_{1,0}, U_{r}=L_{0}$ and $U_{i}=L_{i, 0}$ for $i=2, \ldots, r-1$; this shows that $\mathbf{U}$ satisfies the condition $U_{i} \cap U_{j}=\{0\}$ for any two distinct indices $i$ and $j$. By Lemma 4.8, each $U_{i}$ $(i=2, \ldots, r-1)$ is a subgroup of $K$. Thus $\mathbf{U}$ is indeed a PCP. Constructing from $\mathbf{U}$ the associated mappings $H \rightarrow G$ as in the proof of Proposition 4.1 just gives the $\varphi_{i}$ we started with. Since $G$ is a normal subgroup of $K$, the translation net belonging to $\mathbf{U}$ and the net $\mathbf{N}$ belonging to $\Phi$ coincide (as we have seen in the proof of Proposition 4.5). Conversely, let $\mathbf{N}$ be any translation $(s, r)$-net with translation group $K$ and a transitive direction with corresponding group $G$ of central translations. By Proposition 4.6, we may assume that $G$ is a normal subgroup of $K$. Let $\mathbf{U}$ be the corresponding PCP and proceed as in the proof of 4.1 to obtain a system $\Phi=\left\{\varphi_{2}, \ldots, \varphi_{r-1}\right\}$ of pairwise orthogonal $(G, H)$-orthomorphisms from $H$ onto $G$. As $G$ is a normal subgroup of $K$ with complement $H$, we may identify $K$ with the semi-direct product of $G$ by $H$ for the suitable homomorphism $\pi$ from $H$ into Aut $G$ (in fact, $\pi_{h}$ is the conjugation by $h$ restricted to $G$ ). By the definition of the $\varphi_{i}$, we then have $U_{i}=\left\{\left(h, \varphi_{i}(h)\right): h \in H\right\}$ for $i=2, \ldots, r-1$. Once more using Lemma 4.8 , we see that all $\varphi_{i}$ are indeed (pairwise orthogonal) semiisomorphisms.

We mention that 4.9 also provides a proof of parts (b) and (c) of Theorem 3.5, since $U_{i}(i=2, \ldots, r-1)$ is isomorphic to $H=U_{1}$ by Lemma 4.9.

In view of the preceding arguments, we shall call a translation net with (at least) one transitive direction a semi-splitting translation net. Then the previous results may be combined to obtain the following characterization theorem:

4.10 THEOREM. The following are equivalent concepts:

(a) semi-splitting translation nets;

(b) PCPs with a normal component;

(c) systems of pairwise orthogonal semi-isomorphisms.

We now specialize our results to the case of direct products. Then we have 
$\pi_{h b}=\mathrm{id}_{G}$ for all $h \in H$. and thus our pairwise orthogonal semi-isomorphisms $H \rightarrow G$ become pairwise orthogonal isomorphisms. But then we may as well replace $H$ by the isomorphic group $G$ and the isomorphisms become pairwise orthogonal fixed-point-free automorphisms of $G$. Together with the results of Section 3. we obtain from 4.10 the following result:

\subsection{THEOREM. The following are equivalent concepts:}

(a) Bruck nets with two transitive directions;

(b) splitting translation nets:

(c) PCPs with two normal components;

(d) systems of pairwise orthogonal fixed-point-free group automorphisms.

The equivalence of parts (b), (c) and (d) of Theorem 4.11 has already been shown by Bailey and Jungnickel [2] using different arguments. This result generalizes the well-known result that a system of $s-2$ pairwise orthogonal orthomorphisms of a group $G$ of order $s$ describes an affine translation plane if and only if these orthomorphisms are in fact fixed-point-free group automorphisms. This result is due to Repphun [26] and has recently been rediscovered by Evans [9]. The main results of Evans' paper concern the derivation of upper bounds on the number of pairwise orthogonal fixedpoint-free group automorphisms. In view of Theorem 4.11, this question is equivalent to bounds on the maximum number of components in a PCP describing a splitting translation net. Thus most of his results in this direction are special cases of corresponding bounds in [19] which hold for translation nets in general. Moreover, in the special case of splitting translation nets one can obtain much stronger results (see [2]); in particular, these authors determine the exact maximum number of components of a splitting translation net, i.e. of pairwise orthogonal fixed-point-free group automorphisms for every abelian group $G$.

We conclude this section with a few examples of semi-isomorphisms which are not isomorphisms:

4.12 EXAMPLE. Let $G=\mathbf{Z}_{p^{2}}$ be the cyclic group of residues $\bmod p^{2}$, where $p$ is an odd prime. We define an automorphism $\pi_{1}$ of $G$ by $\pi_{1}(x)=(p+1) x$. Note that an easy induction shows

$$
\pi_{n}(x):=\pi_{1}\left(\pi_{n-1}(x)\right)=(p+1)^{n} x=(n p+1) x
$$

for all $x \in G$. In view of $\left({ }^{*}\right)$, the mapping $\pi: G \rightarrow$ Aut $G$ with $\pi: n \rightarrow \pi_{n}$ is a homomorphism and therefore defines a semi-direct product $K$ of $G$ by itself; we leave it to the reader to show that $K$ is the metacyclic group of order 
$p^{4}$ (cf. Huppert [11, III.12.6]). We now define $p-1$ mappings $\varphi_{i}: G \rightarrow G$ $(i=1, \ldots, p-1)$ by putting

$$
\left({ }^{* *}\right) \quad \varphi_{i}(y):=i y+\frac{p i y(y-1)}{2} ;
$$

we claim that these mappings are semi-isomorphisms of $G$ onto itself. We first show that they are bijections. Thus assume $\varphi_{i}(y)=\varphi_{i}(z)$, i.e. $i(y-z)=$ pi $\left(z^{2}-z-y^{2}+y\right) / 2$. Then $p$ has to divide $y-z$ and thus also $y^{2}-z^{2}$; it follows that $p$ divides $z^{2}-z-y^{2}+y$ and therefore $p^{2}$ divides $y-z$ (since $i$ is not divisible by $p$ ), which shows that $y=z$ in $G$. It remains to show that $\varphi_{i}$ satisfies condition (4.4). But $\left({ }^{*}\right)$ and $\left({ }^{* *}\right)$ give

$$
\begin{aligned}
\varphi_{i}\left(y+y^{\prime}\right) & =i\left(y+y^{\prime}\right)+\frac{p i\left(y+y^{\prime}\right)\left(y+y^{\prime}-1\right)}{2} \\
& =i y+\frac{p i y\left(y+2 y^{\prime}-1\right)}{2}+i y^{\prime}+\frac{p i y^{\prime}\left(y^{\prime}-1\right)}{2} \\
& =\left(p y^{\prime}+1\right)\left(\frac{i y+p i y(y-1)}{2}\right)+i y^{\prime}+\frac{p i y^{\prime}\left(y^{\prime}-1\right)}{2} \\
& =\pi_{y^{\prime}}\left(\varphi_{i}(y)\right)+\varphi_{i}\left(y^{\prime}\right) .
\end{aligned}
$$

It is easily checked that the $\varphi_{i}$ are pairwise orthogonal. Thus there exists a $\left(p^{2}, p+1\right)-\mathrm{PCP}$ with a normal component in $K$. We remark that such a PCP has already been constructed explicitly in the metacyclic group of order $p^{4}$ in [16].

4.13 EXAMPLE. We form a semi-direct product $K$ of $G=\mathbf{Z}_{6}$ by $H=S_{3}$, where we write $S_{3}=\left\langle a, b: a^{3}=b^{2}=1, b a b=a^{2}\right\rangle$ multiplicatively. For $\pi: H \rightarrow$ Aut $G$ we choose the homomorphism defined by $\pi_{a}=\mathrm{id}_{G}$ and $\pi_{b}: z \rightarrow 5 z$. Then the mapping $\varphi$ defined by

$$
1 \rightarrow 0, \quad a \rightarrow 2, \quad a^{2} \rightarrow 4, \quad b \rightarrow 3, \quad b a \rightarrow 5, \quad b a^{2} \rightarrow 1
$$

is a semi-isomorphism. This may be checked by direct verification. This example is adapted from a PCP given by Sprague [30]. We leave the readers to convince themselves that it is also possible to form a semi-direct product of $G=S_{3}$ by $H=\mathbf{Z}_{6}$ and to find a semi-isomorphism in this case, too.

\section{Class regular and translation transversal designs}

In this section, we consider the dual version of Bruck nets with a transitive direction, i.e. 'class regular' transversal designs, and their connection to the translation transversal designs studied by Schulz [27]-[29] and Biliotti and 
Micelli [4]. The correspondence between right difference matrices and class regular TDs was the original way of giving a geometrical interpretation of difference matrices (cf. [2]. [14]. [18]). We first recall the definition of class regularity:

5.1 DEFINITION. Let $G$ be a collineation group of a transversal design D. Then $\mathbf{D}$ is called class regular with respect to $G$ if $G$ fixes the point classes of $\mathbf{D}$ and acts regularly on each of the point classes.

It is easily seen that a class regular TD is resolvable (with the orbits of $G$ on the lines of $\mathbf{D}$ as parallel classes); thus $\mathbf{D}$ may be extended to a larger TD by adding a further point class $P$. Note that $G$ will fix every point in this new point class. When dualizing the notion of an $(s, r+1)$-net $\mathbf{N}$ with a transitive direction $\mathbf{P}$, we obtain a class regular $\mathrm{TD}[r, s]$ corresponding to the $s$ parallel classes $\neq \mathbf{P}$ of $\mathbf{N}$; then the new point class $P$ that may be added corresponds to $\mathbf{P}$. We refer the reader to [3] for details on class regular TDs. The following result may be considered as the dual version of Theorem 2.3.

5.2 THEOREM. The existence of an $(s, r)$-difference matrix over $G$ is equivalent to that of a class regular $\mathrm{TD}[r, s]$ (with respect to $G$ ).

Proof. Since the proof is similar to that of Theorem 2.3 and may be found in [3] or [14], we shall only sketch the construction of the TD from the difference matrix. (Note that one now needs to use right difference matrices if $G$ is supposed to act on the TD from the right.) Thus let $D$ be a right $(s, r)$ difference matrix over $G$ and define an incidence structure $\mathbf{D}$ as follows. The point set $V$ consists of all pairs $(i, g)$ with $i=1, \ldots, r$ and $g \in G$. As blocks one chooses the sets $B_{j, y}:=\left\{\left(i, d_{i j}+y\right): i=1, \ldots, r\right\}$ where $j=1, \ldots, s$ and $y \in G$.

5.3 REMARK. As in Lemma 2.5, we may identify a normalized right ( $s, r)$ difference matrix $D$ with a system of $r-2$ pairwise orthogonal right orthomorphisms $\varphi_{3}, \ldots, \varphi_{r}$ of $G$. Let $\varphi_{1}$ and $\varphi_{2}$ be defined by $\varphi_{1}(g)=0$ and $\varphi_{z}(g)=y$. respectively, for all $g \in G$. Of course, this again corresponds to indexing the columns of $D$ with the elements in the second row. In analogy to Remark 2.6, we may then give the following alternative description of the TD D associated with $D$ : Put $\Phi=\left\{\varphi_{1}, \ldots, \varphi_{r}\right\}$ and $V=\Phi \times G$. Then the blocks are the sets

$$
B_{x, y}=\{(\varphi, g) \in V: g=\varphi(x)+y\}, \text { where }(x, y) \in G \times G .
$$

The point classes are the sets $P_{\varphi}=\{(\varphi, g): g \in G\}$ (with $\left.\varphi \in \Phi\right)$ and $z \in G$ acts on D as follows:

$$
(\varphi, g) \rightarrow(\varphi, g+z) \text { and } \quad B_{x, y} \rightarrow B_{x, y+z}
$$


We shall now investigate under which conditions a class regular TD D is a 'translation' TD. Let us first recall the required definitions and basic results as introduced by Schulz ([28], [29]).

5.4 DEFINITION. Let $K$ be a collineation group of a resolvable transversal design $\mathbf{D}$ which acts regularly on the set of points and fixes each parallel class. Then $\mathbf{D}$ is called a translation transversal design (for short, a TTD) with translation group $K$.

5.5 DEFINITION. Let $K$ be a group of order $r s$. Then a set $\mathbf{U}=\left\{G, U_{1}, \ldots, U_{s}\right\}$ of pairwise disjoint subgroups of $K$ is called an $(s, r)$ partition of $K$ if it satisfies the following conditions:

(5.1) $|G|=s$ and $\left|U_{i}\right|=r$ for $i=1, \ldots, s$.

(5.2) Each $k \in K$ is contained in a component of $\mathbf{U}$ (which is, of course, unique if $k \neq 0$ ).

We also call $G$ the special component of $\mathbf{U}$.

One now has the following analogue of Proposition 3.4:

5.6 PROPOSITION. Let $\mathbf{U}$ be an $(s, r)$-partition of the group $K$. Then the incidence structure

$$
\left.\mathbf{D}=\mathbf{D}(\mathbf{U})=\left(K,{ }_{i} U_{i}+g: i=1, \ldots, s ; g \in G\right\}, \epsilon\right)
$$

is an translation $\mathrm{TD}[r, s]$ with translation group $K$; the point classes of $\mathbf{D}$ are the right cosets of $G$. Moreover, every translation TD may be represented in this way.

The proof of 5.6 will be left to the reader; it may be found in [4] and [29]. There is a well-developed theory of TTDs; they have been classified based on the classification of finite groups with a partition. Many series of examples have been constructed. We refer the reader to Biliotti and Micelli [4] and to Schulz [27]-[29].

We next show the following analogue of Proposition 4.1:

5.7 PROPOSITION. Let $G$ be the special component of an $(s, r)$-partition $\mathbf{U}$ of the group $K$. Then there exists an $(s, r)$-difference matrix over $G$.

Proof. Let $\mathbf{U}=\left\{G, U_{1}, \ldots, U_{s}\right\}$; we shall give an explicit construction for a right ( $s, r)$-difference matrix $D$ over $G$. Put $H=U_{1}$ and note that every element $k \in K$ has a unique representation $k=h+g$ with $g \in G$ and $h \in H$. In particular, every $u \in U_{i}(i=1, \ldots, s)$ has a unique representation in the form $u=h+\varphi_{h}(i)$ with $h \in H$ and $\varphi_{h}(i) \in G$. As each $U_{i}$ is a system of coset representatives for $G$, this defines $r$ mappings $\varphi_{h}:\{1, \ldots, s\} \rightarrow G(h \in H)$. We now construct a matrix 
$D=\left(d_{h i}\right)_{h \in H: i=1 \ldots . . s}$ over $G$ by putting $d_{h i}:=\varphi_{h}(i)$ and claim that $D$ is a right difference matrix. To see this, consider two distinct column indices $i$ and $j$ and assume that

$$
\varphi_{h}(i)-\varphi_{k}(i)=\varphi_{h}(j)-\varphi_{k}(j) \text { for } h, k \in H:
$$

we have to show that this implies $h=k$. By definition of the $\varphi_{h}(i)$, there are elements $u, u^{\prime} \in U_{i}$ and $\quad v, v^{\prime} \in U_{j} \quad$ satisfying $h+\varphi_{h}(i)=u, \quad k+\varphi_{k}(i)=u^{\prime}$, $h+\varphi_{h}(j)=v$ and $k+\varphi_{k}(j)=v^{\prime}$. Thus $\left(^{*}\right)$ implies $(-h+u)-\left(-k+u^{\prime}\right)=$ $(-h+v)-\left(-k+v^{\prime}\right)$ and thus $u-u^{\prime}=v-v^{\prime} \in U_{i} \cap U_{j}=\{0\}$ which gives $u=u^{\prime}$ and $v=v^{\prime}$. We now have $h+\varphi_{h}(i)=u=k+\varphi_{k}(i)$ and thus $\varphi_{h}(i)-\varphi_{k}(i)=-h+k \in G \cap H=\{0\}$ which finally proves that indeed $h=k$.

As in Section 4, one can show that the TDs belonging to $\mathbf{U}$ and $D$, respectively, coincide if and only if $G$ is a normal subgroup of $K$. We leave this to the reader and now consider translation TDs which are also class regular. The following result is similar to Proposition 4.6:

5.8 PROPOSITION. Let $\mathbf{D}$ be a translation TD with translation group $K$ and assume that $\mathbf{D}$ is also class regular with respect to a group $G^{\prime}$. Then $\mathbf{D}$ is also a translation TD for some translation group $K^{\prime}$ containing $G^{\prime}$ as a normal subgroup.

Proof. Since the arguments required are similar to those used in Sections 2 and 4 , we shall only sketch them. Let $\mathbf{U}=\left\{G, U_{1}, \ldots, U_{s}\right\}$ be the $(s, r)$ partition of $K$ describing $D$. Note that both $G$ and $G^{\prime}$ fix the point class $G$. In the case $G=G^{\prime}$, there is nothing to prove. Thus assume $G \neq G^{\prime}$. One first proves an analogue of Proposition 2.7, i.e. that any translation of $\mathbf{D}$ which fixes each point class is uniquely determined by the image of a single point $p$. Then let $\alpha$ and $\beta$ be arbitrary elements of $K$ and $G^{\prime}$, respectively, and check that $\alpha^{-1} \beta \alpha$ fixes each point class of $\mathbf{D}$ and is either fixed-point-free or the identity. Using this, one proves that $U_{1}$ normalizes $G^{\prime}$, and thus the group $K^{\prime}$ generated by $G^{\prime}$ and $U_{1}$ is the semi-direct product of $G^{\prime}$ by $U_{1}$. It is then easy to see that $K^{\prime}$ acts regularly on the points of $\mathbf{D}$, which shows that $\mathbf{D}$ is also a translation TD with respect to $K^{\prime}$.

With the notation of the preceding theorem, the $(s, r)$-partition of the group $K^{\prime}$ describing $\mathbf{D}$ will of course have $G^{\prime}$ as its special component. In view of Proposition 5.8, we therefore need only consider translation TDs represented by an $(s, r)$-partition $U$ in a group $K$ for which the special component $G$ is a normal subgroup of $K$ when we want to study TTDs which are simultaneously class-regular. We remark that we do not know whether there exist any $(s, r)$-partitions for which the special component is not a normal subgroup. Note that $K$ is a semi-direct product of $G$ by $H$ (where $H$ is any 
other component of $\mathbf{U}$ ) if the special component $G$ is normal. Thus we have to study $(s, r)$-partitions in suitable semi-direct products. The following result characterizes such $(s, r)$-partitions; this is somewhat similar to Proposition 4.9.

5.9 PROPOSITION. Let $G$ and $H$ be two groups of orders $s$ and $r$, respectively. Also, let $\pi: H \rightarrow$ Aut $G$ be a homomorphism and denote the corresponding semi-direct product of $G$ by $H$ as $K$. Then the following two assertions are equivalent:

(a) There exists an (s,r)-partition $\mathbf{U}$ of $K$ with $\hat{G^{\prime}}=\{0\} \times G$ as special component and $\mathrm{H}^{\wedge}=H \times\{0\}$ as a further component.

(b) There exists a system $\Psi=\left\{\psi_{g}: g \in G\right\}$ of semi-homomorphisms from $H$ into $G$ (cf. Definition 4.7) satisfying the following condition:

(5.3) The mapping $h \rightarrow-\psi_{g}(h)+\psi_{f}(h)$ is injective for all $f, g \in G$ with $f \neq g$.

Proof. First let $\mathbf{U}=\left\{G, U_{1}, \ldots, U_{s}\right\}$ be an $(s, r)$-partition of $K$ with $H=U_{1}$ (where we abuse notation and identify $G$ with $G^{\wedge}$ and $H$ with $H^{\wedge}$ ). We construct the mappings $\varphi_{h}:\{1, \ldots, s\} \rightarrow G(h \in H)$ as in the proof of Proposition 5.7; as we have seen, these mappings define a normalized right $(s, r)$-difference matrix. We may therefore change notation and index the components of $\mathbf{U}$ by using the elements of $G$ as index set (instead of the set $\{1, \ldots, s\}$ ); then the $\varphi_{h}$ become (pairwise orthogonal) mappings from $G$ into itself. We now define $s$ mappings $\psi_{g}: H \rightarrow G$ by putting $\psi_{g}(h):=\varphi_{h}(g)$. Using this notation and the definition of the $\varphi_{h}$, we have

$$
U_{g}=\left\{h+\varphi_{h}(g): h \in H\right\}=\left\{h+\psi_{g}(h): h \in H\right\}=\left\{\left(h, \psi_{g}(h)\right): h \in H\right\} .
$$

Now $U_{g}$ is closed under addition; in view of (4.5), this shows the validity of (4.4) which means that the $\psi_{g}$ are semi-homomorphisms. It remains to check the validity of (5.3). Thus we want to show

$-\psi_{g}(h)+\psi_{f}(h)=-\psi_{g}(k)+\psi_{f}(k) \Rightarrow h=k \quad$ (with $h, k \in H$ and $f, g \in G, f \neq g$ );

but this is equivalent to

$-\varphi_{h}(g)+\varphi_{h}(f)=-\varphi_{k}(g)+\varphi_{k}(f) \Rightarrow h=k \quad($ with $h, k \in \underline{H}$ and $f, g \in G, f \neq g$ ).

Reordering terms, we want to show

$\varphi_{h}(g)-\varphi_{k}(g)=\varphi_{h}(f)-\varphi_{k}(f) \Rightarrow h=k \quad$ (with $h, k \in H$ and $f, g \in G, f \neq g$ )

which is true since the $\varphi_{h}$ are pairwise orthogonal (as shown in the proof of Proposition 5.7).

The converse is similar but simpler. Thus let $\Psi=\left\{\psi_{g}: g \in G_{\}}\right.$be a system of 
semi-homomorphisms from $H$ into $G$ satisfying condition (5.3). Put $U_{g}=\left\{\left(h, \psi_{g}(h)\right): h \in H\right\}$ (for all $\left.g \in G\right)$ and observe that the $U_{g}$ are subgroups of $K$ which intersect $G$ trivially: this follows from Lemma 4.8 , since the $\psi_{g}$ are semi-homomorphisms. Using (5.3), it is easily checked that one has $U_{g} \cap U_{f}=\{(0,0)\}$ whenever $f \neq g$. Hence $\mathbf{U}=\left\{G, U_{1}, \ldots, U_{s}\right\}$ is the desired (s. $r)$-partition of $K$.

We note the following consequences of Proposition 5.9 which are analogous to parts (b) and (c) of Proposition 3.5:

5.10 COROLlaRY. Let $\mathbf{U}=\left\{G, U_{1}, \ldots, U_{s}\right\}$ be an $(s, r)$-partition in $K$, where the special component $G$ is a normal subgroup. Then the remaining components of $\mathbf{U}$ are pairwise isomorphic.

Proof. We put $H=U_{1}$; then $K$ is a semi-direct product of $G$ by $H$ and we may use the results of Proposition 5.9. Thus we have the system $\Psi=\left\{\psi_{g}: g \in G\right\}$ of semi-homomorphisms from $H$ into $G$; moreover, the components of $\mathbf{U}$ can be written as $U_{g}=\left\{\left(h, \psi_{g}(h)\right): h \in H\right\}$. Now Lemma 4.8 shows that $U_{g} \cong H$.

5.11 COROLlaRY. Let $\mathbf{U}=\left\{G, U_{1}, \ldots, U_{s}\right\}$ be an $(s, r)$-partition in $K$, where both the special component $G$ and a further component (say, $\left.H:=U_{1}\right)$ are normal subgroups. Then all $U_{i}$ are isomorphic to a subgroup $H^{\prime}$ of $G$, and $K \cong G \times H$. Moreover, the system $\Phi=\left\{\varphi_{h}: h \in H\right\}$ of mappings constructed as in the proof of Proposition 5.7 forms a group (isomorphic to $H$ ) with respect to pointwise addition.

Proof. In view of Corollary 5.10, the components $U_{i}$ are pairwise isomorphic. As in the proof of Proposition 5.9, we relabel the components as $U_{g}$ by using the elements of $G$ as indices. Since $K$ is now the direct product of the components $G$ and $H$, the homomorphism $\pi$ from $H$ into Aut $G$ is trivial and all the semi-homomorphisms $\psi_{g}$ are in fact homomorphisms. Note that we have $\psi_{0}(g)=\varphi_{g}(0)=0$ for all $g \in G$ (by construction of the $\varphi_{h}$ ). Then condition (5.3) shows that all $\psi_{g}$ with $g \neq 0$ are injective; thus each $U_{g}$ is isomorphic to a subgroup of $G$. It only remains to check the last assertion; thus let $h, k \in H$ and $g \in G$. Then we have

$\varphi_{h+k}(g)=\psi_{g}(h+k)=\psi_{g}(h)+\psi_{g}(k)=\varphi_{h}(g)+\varphi_{k}(g)=\left(\varphi_{h}+\varphi_{k}\right)(g)$

which shows that the $\varphi_{h}$ form a group isomorphic to $H$ under pointwise addition.

Corollary 5.11 admits the following converse:

5.12 PROPOSITION. Let $G$ and $H$ be groups of order $s$ and $r$, respectively, and let $\Phi=\left\{\varphi_{h}: h \in H\right\}$ be a system of mappings from $G$ into itself, where 
$\varphi_{0}(g)=0$ for all $g \in G, \varphi_{1}(g)=g$ for all $g \in G$ (where 1 denotes a fixed element $\neq 0$ of $H$ ) and where the $\varphi_{h}$ with $h \neq 0,1$ are pairwise orthogonal right orthomorphisms of $G$. Assume that $\Phi$ satisfies

$$
\varphi_{h}+\varphi_{k}=\varphi_{h+k} \quad \text { for all } h, k \in H,
$$

i.e $\Phi$ is a group isomorphic to $H$ under pointwise addition. Then there exists an $(s, r)$-partition $\mathbf{U}$ with special component $G$ and a further component $H$ in the direct product $K=G \times H$.

Proof. Put $U_{g}:=\left\{\left(h, \varphi_{h}(g)\right): h \in H\right\}$; then (5.4) implies that the $U_{g}$ are subgroups of $K$. Using the fact that the $\varphi_{h}$ are pairwise orthogonal, one easily checks that the $U_{g}$ are pairwise disjoint. Hence $\mathbf{U}=\{G\} \cup\left\{U_{g}: g \in G\right\}$ is the desired $(s, r)$-partition of $K$.

We shall call a TTD described by an $(s, r)$-partition as in Corollary 5.11 and Proposition 5.12 a splitting TTD. Combining the previous results, we get the following characterization:

5.13 THEOREM. The following are equivalent concepts:

(a) splitting translation TDs:

(b) sistems of group homomorphisms satisfying condition (5.3);

(c) difference matrices whose rows form a group under pointwise addition.

Theorem 5.13 generalizes the fact that dual translation planes and complete systems of orthomorphisms forming together with the 0-mapping and the identity a group under pointwise addition are equivalent; this result is due to Repphun [26] and has been rediscovered by Evans [9]. We close this section with a few examples of splitting TTDs. Clearly the multiplication table of $\mathrm{GF}\left(p^{a}\right), p$ a prime, is a difference matrix whose rows form a group $H$ under pointwise addition; this example belongs to the affine plane $\operatorname{AG}\left(2, p^{a}\right)$ which may be considered as a splitting TTD by removing any parallel class of lines. Taking any proper subgroup of $H$ yields further examples. We mention a class of non-abelian examples:

5.14 EXAMPLE. Let $G$ be any non-abelian $p$-group of order $p^{a}$ with exponent $p$, let $H=Z_{p}$ and define $K:=G \times H$. Then $K$ is a non-abelian $p$ group of order $p^{a+1}$ with exponent $p$. Then the set of all subgroups $\langle x\rangle$, where $x$ runs over the elements of $K \backslash G$, forms together with $G$ a $\left(p^{a}, p\right)$ partition of $K$ with the normal components $G$ and $H$.

\section{EXTENSIONS}

In this final section. we consider the problem of extending an $(s, r)$-net with a 
transitive direction to an $(s, r+1)$-net. The following result is essentially due to Ostrom [23] and has also been considered in [3] (where a slight mistake has been made by using right difference matrices instead of left difference matrices).

6.1 THEOREM. Let $D$ be a left $(s, r-1)$-difference matrix over $G$ and let $\mathbf{N}$ be the associated (s.r)-net with transitive direction $\mathbf{P}_{0}$ (as in Theorem 2.3). Then the following conditions are equivalent:

(a) $\mathbf{N}$ has a 'transversal' (i.e. a set of s points meeting each line in a unique point).

(b) $\mathbf{N}$ can be extended to an $(s, r+1)$-net $\mathbf{N}^{\prime}$ with transitive direction $\mathbf{P}_{0}$.

(c) $D$ can be extended to an $(s, r)$-difference matrix over $G$ by adding a suitable row.

Proof. In view of Theorem 2.3, conditions (b) and (c) are obviously equivalent. Now let $d_{r j}(j=1, \ldots, s)$ be any $s$ elements of $G$ and put $T:=\left\{\left(j, d_{r j}\right): j=1, \ldots, s\right\}$. Then $T$ intersects each of the lines $L_{j}$ uniquely. Since $L_{i, x}=\left\{\left(j, d_{i j}+x\right): j=1, \ldots, s\right\}$, the intersection points of $T$ with $L_{i, x}$ correspond to the solutions of the equation

$$
-d_{r j}+d_{i j}=-x
$$

Thus $T$ is a transversal for $\mathbf{N}$ if and only if $\left({ }^{*}\right)$ has - for any given $x$ and any given $i-$ a unique solution for $j$ if and only if the row $\left(d_{r 1}, \ldots, d_{r s}\right)$ extends $D$ to an $(s, r)$-difference matrix.

6.2 COROLLARY. Let $\mathbf{N}$ be a Bruck net with a transitive direction $\mathbf{P}$. Then every transversal is contained in a Bruck net $\mathbf{N}^{\prime}$ with the same transitive direction $\mathbf{P}$.

Note, however, that Corollary 6.2 does not claim that all extensions of $\mathbf{N}$ have $\mathbf{P}$ as a transitive direction. It is still conceivable that a clever choice of transversals results in a parallel class which is not fixed under the group $G$ of central translations of $\mathbf{N}$ with direction $\mathbf{P}$. In fact, a construction due to Dow [8] provides an infinite series of examples which we shall now sketch. We refer the reader to Hughes and Piper [10] for the required results on affine planes.

6.3 EXAMPLE. Let $q$ be a prime power and consider the $\left(q^{2}, q^{2}-q\right)$-net $\mathbf{N}$ which consists of the parallel classes in common to the Desarguesian plane $\mathrm{AG}\left(2, q^{2}\right)$ and its derived plane, the Hall plane $\mathrm{H}\left(q^{2}\right)$. Then one may construct a parallel class $\mathbf{T}$ of transversals of $\mathbf{N}$ which contains lines from the missing parallel classes of both these planes. As the lines of $\mathrm{H}\left(q^{2}\right)$ not in $\mathrm{AG}\left(2, q^{2}\right)$ are 
Baer subplanes of $\mathrm{AG}\left(2, q^{2}\right)$, we may also say that we can form $\mathbf{T}$ by selecting some of the missing lines of $\mathrm{AG}\left(2, q^{2}\right)$ together with some Baer subplanes. Since the translations of $\mathbf{N}$ are just those of $\mathrm{AG}\left(2, q^{2}\right)$, it is then clear that the extension $\mathbf{N}^{\prime}$ of $\mathbf{N}$ obtained by adjoining $\mathbf{T}$ cannot be invariant under the translation group of $\mathbf{N}$. Using results of Ostrom [22], one may show that $\mathbf{N}^{\prime}$ is then transversal-free. We refer the reader to Dow [8] for details.

However, if $r$ is sufficiently large, then every extension of $\mathbf{N}$ will still have transitive direction $\mathbf{P}$. Recall that an $(s, r)$-net is said to have small deficiency if the deficiency $d:=s+1-r$ satisfies the inequality $s>(d-1)^{2}$, i.e. $d \leqslant \sqrt{s}$ if $s$ is a square. Note that the examples given in 6.3 have $d=1+\sqrt{s}$. Thus the following result is best possible:

6.4 THEOREM. Let $\mathbf{N}$ be a Bruck net with transitive direction $\mathbf{P}$. If $\mathbf{N}$ has small deficiency, then every extension of $\mathbf{N}$ also has transitive direction $\mathbf{P}$.

Proof. As $\mathbf{N}$ has small deficiency, we have the following result due to Bruck [6]: Any two distinct transversals of $\mathbf{N}$ have at most one point in common. (A proof of this may also be found in [3].) Now let $T$ be a transversal of $\mathbf{N}$ and denote the transitive group of central translations with direction $\mathbf{P}$ by $G$. By Corollary 6.2, the orbit of $T$ under $G$ is a parallel class $\mathbf{T}$ of transversals of $\mathbf{N}$. Assume that $T$ is also contained in another parallel class $\mathbf{S}$ of transversals of $\mathbf{N}$. Then any other transversal $S \in \mathbf{S}$ has to meet at least one of the $s-1$ transversals in $\mathbf{T}$ which are different from $T$ in more than one point, a contradiction.

We note the following consequence of Theorem 6.4 which improves results of Jungnickel [17].

6.5 THEOREM. Let $\mathbf{N}$ be a translation net of small deficiency with translation group $K$, and assume that $\mathbf{N}$ has order $s \neq 4$. Then any extension $\mathbf{N}^{\prime}$ of $\mathbf{N}$ is also a translation net with translation group $K$. Moreover, the PCP describing $\mathbf{N}^{\prime}$ is obtained from that describing $\mathbf{N}$ by adding a further component.

Proof. Assume first that $\mathbf{N}$ has order $s \neq 2$ or 4 . Then $\mathbf{N}$ has elementary abelian translation group $K$ by a result of [17] and thus all directions of $\mathbf{N}$ are transitive by Proposition 3.5. Then the assertion is an easy consequence of Theorem 6.4. For $s=2$, the condition $r \geqslant 3$ ensures that $\mathbf{N}$ is the affine plane of order 2 and therefore not extendable.

6.6 COROLLARY. Let $\mathbf{N}$ be a translation net of small deficiency with translation group $K$, described by the PCP $\mathrm{U}$. Then the following conditions are equivalent: 
(a) $\mathbf{N}$ has no transversals;

(b) $\mathbf{N}$ is maximal;

(c) $\mathbf{U}$ is maximal.

In [17] it was shown that certain PCPs constructed by Bruen [7] belong to translation nets of order $q^{2}$ and deficiency $q$ which cannot be embedded into an affine plane. Recently, Johnson [13] has constructed PCPs of order $q^{2}$ and deficiency $q-1$ for prime powers $q \equiv 2(\bmod 3)$ which also lead to nonimbeddable (translation) nets. It is an open problem whether or not these nets are maximal if $q$ is not a prime (in which case they are indeed maximal by a result of [17]). While we cannot solve this problem, Theorem 6.5 shows that any extension would necessarily be a translation net (obtained by adding further components to the PCP).

We close this section with an example showing that the assumption $s \neq 4$ in Theorem 6.5 is indeed necessary.

6.7 EXAMPLE. Let $K:=\left\langle a, b, c: a^{2}=b^{2}=c^{4}=1, c^{-1} a c=b, c^{-1} b c=a\right\rangle$. Thus $K$ is a (multiplicatively written) non-abelian group of order 16 , i.e. the semi-direct product of the elementary abelian group of order 4 by the cyclic group of order 4 . Then the components

$$
U_{1}=\langle c\rangle, \quad U_{2}=\langle a, b\rangle \text { and } U_{3}=\langle a c\rangle
$$

form a (4, 3)-PCP $\mathbf{U}$ in $K$. Note that $\mathbf{U}$ is a maximal PCP; this follows from [16] but is also easily checked directly. By Lemma 4.2 of [17], the corresponding net $\mathbf{N}$ is either transversal-free or can be extended to the affine plane $\mathbf{A}=\mathbf{A G}(2,4)$. In fact, the latter possibility holds; the reader may easily check that $S=\left\{1, b c, b c^{2}, a b c^{3}\right\}$ and $T=\left\{1, a b c, a c^{2}, a c^{3}\right\}$ are two transversals and that the orbits of $S$ and $T$ under $U_{2}$ are the two missing parallel classes which complete $\mathbf{N}$ to $\mathbf{A}$. Of course, $\mathbf{A}$ is also a translation net, but not with respect to $K$. Note that $\mathbf{N}$ is a further example of a translation net with translation group $K$ and a transitive group $G$ of central translations which is not contained in $K$. We finally comment on the geometric significance of $K$ : If one considers $\mathbf{N}$ embedded into the projective plane $\operatorname{PG}(2,4)$, then the elements of order 4 in $K$ are 'generalized elations', as studied, e.g., by Vedder $[31]$.

\section{REFERENCES}

1. André, J., 'Über nicht-Desarguessche Ebenen mit transitiver Translationsgruppe’, Math. $Z$. 60 (1954), 156-186.

2. Bailey, R. A. and Jungnickel, D., 'Translation nets and fixed-point-free group automorphisms' (J. Combin. Theory Ser. A, in press). 
3. Beth, T., Jungnickel, D. and Lenz, H., Design Theory, Cambridge Univ. Press, 1986.

4. Biliotti, M. and Micelli, G., 'On translation transversal designs', Rend. Sem. Mat. Univ. Padova 73 (1985), 217-229.

5. Bose, R. C. and Bush, K. A., 'Orthogonal arrays of strength two and three', Ann. Math. Stat. 23 (1952), 508-524.

6. Bruck, R. H., 'Finite nets II. Uniqueness and embedding', Pacific J. Math. 13(1963), 421-457.

7. Bruen, A. A., 'Unimbeddable nets of small deficiency', Pacific J. Math. 43 (1972), 51-54.

8. Dow, S., 'Transversal-free nets of small deficiency', Arch. Math. 41 (1983), 472-474.

9. Evans, A. B., 'Orthomorphism graphs of groups', J. Geom. 35 (1989), 66-74.

10. Hughes, D. R. and Piper, F. C., Projective Planes, Springer, Berlin, Heidelberg, New York, 1973.

11. Huppert, B., Endliche Gruppen I, Springer, Berlin, Heidelberg, New York, 1967.

12. Johnson, D., Dulmage, A. M. and Mendelsohn, N. S., 'Orthomorphisms of groups and orthogonal Latin squares', Canad. J. Math. 13 (1961), 356-372.

13. Johnson, N. L., 'Maximal partial spreads and central groups' (Preprint).

14. Jungnickel, D., 'On difference matrices, resolvable transversal designs, and generalized Hadamard matrices', Math. Z. 167 (1979), 49-60.

15. Jungnickel, D., 'On difference matrices and regular Latin squares', Abh. Math. Sem. Hamburg 50 (1980), 219-231.

16. Jungnickel, D., 'Existence results for translation nets', in Finite Geometries and Designs. London Math. Soc. Lecture Notes 49 (1981), 172-196, Cambridge Univ. Press.

17. Jungnickel, D., 'Maximal partial spreads and translation nets of small deficiency', J. Algebra 90 (1984), 119-132.

18. Jungnickei, D., 'Lateinische Quadrate, ihre Geometrien und ihre Gruppen', Jahresber. $\bar{D} M V$ 86 (1984), 69-108.

19. Jungnickel, D., 'Existence results for translation nets II', J. Algebra 122 (1989), 288-298.

20. Jungnickel, D., 'Latin squares, their geometries and their groups. A survey', in D. RayChaudhuri (ed.). Coding Theory and Design Theory Part II. IMA Vol. 21 (1990), Springer, New York, pp. 166-225.

21. Mann, H. B., 'The construction of orthogonal Latin squares', Ann. Math. Stat. 13 (1942), 418-423.

22. Ostrom, T. G., 'Nets with critical deficiency', Pacific J. Math. 14 (1964), 1381-1387.

23. Ostrom, T. G., 'Replaceable nets, net collineations, and net extensions', Canad. J. Math. 18 (1966), 666-672.

24. Pickert, G., Projektive Ebenen, Springer, Berlin, Heidelberg, New York, 1955.

25. Pickert, G., Einführung in die endliche Geometrie, Klett, Stuttgart, 1974.

26. Repphun, K., 'Geometrische Eigenschaften vollständiger Orthomorphismensysteme von Gruppen', Math. Z. 89 (1965), 206-212.

27. Schulz, R.-H., 'Transversal designs and Hughes-Thompson groups', Mitt. Math. Sem. Giessen 165 (1984), 185-197.

28. Schulz, R.-H., 'Transversal designs and partitions associated with Frobenius groups', $J$. Reine Angew. Math. 355 (1985), 153-162.

29. Schulz, R.-H., 'On the classification of translation group divisible designs', Europ. J. Comb. 6 (1985), 369-374.

30. Sprague, A. P., 'Translation nets', Mitt. Math. Sem. Giessen 157 (1982), 46-68.

31. Vedder, K., 'Generalised elations', Bull. London Math. Soc. 18 (1986), 573-579. 
Authors' address:

Dirk Hachenberger and Dieter Jungnickel, Mathematisches Institut der Justus-Liebig-Universität Gießen,

Arndtstr. 2,

D-6300 Gießen,

F.R.G.

(Received, December 20, 1989) 